GERN-TIS-RP $209 P P$

$C_{1}$

EUROPEAN ORGANIZATION FOR NUCLEAR RESEARCH

26 JUIL. 1988

TIS-RP/209/PP

6 July 1988

\title{
THE INTENSITY DISTRIBUTION OF SECONDARY PARTICLES \\ PRODUCED IN HIGH ENERGY PROTON INTERACTIONS
}

\author{
A.H. Sullivan
}

SUMMARY

An empirical relation is presented that describes the intensity and angular distribution of secondary hadrons around targets struck by high energy protons in an energy range from 5 to $500 \mathrm{GeV}$. This relation gives the fluence of hadrons of energy greater than $40 \mathrm{MeV}$ at 1 metre and at an angle $\theta$ degrees to the direction of a proton of energy $E \mathrm{GeV}$ interacting in a copper target of:

$$
\phi=\frac{1}{3[\theta+(35 / \sqrt{ } \mathrm{E})]^{2}} \text { part } / \mathrm{cm}^{2} / \text { interaction }
$$

It is further shown that if the flux is increased by a factor of 2 the formula also represents well the distribution of neutrons produced in targets struck by protons in the $25 \mathrm{MeV}$ to $2 \mathrm{GeV}$ range. Comparisons are presented between predictions using the above relation and measured and theoretical fluxes, integral particle emission, and dose rate. No serious discrepancies have been found over the entire proton energy range from $25 \mathrm{MeV}$ to $1000 \mathrm{GeV}$. 


\section{INTRODUCTION}

High energy particle interactions produce a range of secondary - hadrons with an intensity and angular distribution that depends strongly on the incident particle energy. An estimate of this secondary hadron flux is necessary as a source term to calculate the dimensions of shields for targets and dumps in proton beams. Furthermore a knowledge of the secondary radiation distribution is required to make predictions of induced radioactivity near targets and of the likelihood of materials sustaining radiation damage.

A number of measurements have been made of the distribution of radiation around targets over a range of proton energies and many theoretical distributions have been computed using Monte Carlo methods. Both the measurements and computations tend to relate to well defined idealized conditions. In order to be able to reliably assess radiation safety problems and to make reasonably realistic predictions of radiation levels near targets and beam dumps, a simple relation is required between the parameters describing secondary radiation from high energy particle interactions. Extrapolations can then be made from data obtained under defined conditions to the actual situations that occur in high energy particle beams. Such a relation should be as simple as possible, need not be exact but should give a reasonable first approximation of the required quantity over a large range of incident proton energies and over the entire angular range.

\section{DISTRIBUTION OF SECONDARY RADIATION INTENSITY FROM PROTON INTERACTIONS}

The secondary radiation intensity from a high energy proton interaction is peaked in the forward direction and at angles greater than about $20^{\circ}$ has been found to decrease by approximately the square of the emission angle $[1,2]$. A study of experimental data of particle fluence versus emission angle from proton interactions at energies in the 5 to $500 \mathrm{GeV}$ range suggests a more universal empirical relation for the secondary hadron fluence at 1 metre from an interaction will be: 


$$
\phi=\frac{1}{3[\theta+(35 / \sqrt{ } E)]^{2}} \text { part } / \mathrm{cm}^{2} / \text { interacting proton }
$$

where $E$ is the proton energy in $\mathrm{GeV}$ and $\theta$ is the angle of emission in degrees.

The particles that have been considered in the analysis are essentially hadrons capable of producing Fluorine-18 in aluminium. This reaction is considered to give a reasonable estimate of flux of hadrons of energy greater than $40 \mathrm{MeV}$.

The flux calculated using equation (1) is compared with experimental data for incident proton energies of $8,24,225$ and 400 $\mathrm{GeV}[3,4]$ on copper targets in Figs. $1 \mathrm{a}$ and $\mathrm{b}$. As can be seen the relation predicts the flux at all energies and angles in the forward direction at which measurements have been made. Measured flux densities in the backward direction from a target risk to be influenced by secondary radiation coming from interactions in vacuum windows and in air and hence are not considered reliable. The relation therefore appears to be reasonable for predicting secondary particle fluxes from high energy particle interactions in any direction and over a large range of proton energies. In particular it allows the secondary particle flux in the forward direction to be estimated which will be given by $\phi_{0}$ where:

$$
\phi_{0}=2.7 \times 10^{-4} \mathrm{E} \text { part } / \mathrm{cm}^{2}
$$

when $E$ is in GeV, and the flux at $90^{\circ}$ will be:

$$
\phi_{90}=\frac{1}{3[90+(35 / \sqrt{ } E)]^{2}} \operatorname{part} / \mathrm{cm}^{2}
$$

These flux densities in the forward and laterial directions to the proton beam are plotted in Fig. 2 as a function of incident proton energy. Apparently for $1 \mathrm{GeV}$ interactions the particle flux at zero degrees is about a factor of 12 higher than that at $90^{\circ}$ while for $100 \mathrm{GeV}$ protons the ratio will be nearer 1000 .

The total number of secondary particles that are emitted per interaction can be calculated by integrating the particle flux given 
by eq. (1) over all angles. This apparent particle multiplicity is shown in Fig. 3 as a function of primary proton energy where it is compared with that computed for proton interactions in iron in the energy range 10 to $1000 \mathrm{GeV}$ using a sophisiticated nuclear interaction model and Monte Carlo methods [5]. The agreement is good at $10 \mathrm{GeV}$ but differs by a factor of 2 at $1000 \mathrm{GeV}$. This discrepancy could possibly be reduced if proper account is taken of the differences in energy threshold above which the flux has been determined in the two cases.

\section{SECONDARY RADIATION DISTRIBUTION FROM PROTON INTERACTIONS OF LESS THAN 2 GEV}

The calculated dependence on proton energy of the number of secondaries produced per interaction was extended down to $10 \mathrm{MeV}$ in Fig. 3. Such an extrapolation is difficult to justify experimentally. In this energy range the secondary radiation of interest is mainly neutrons with a spectrum extending down to below $1 \mathrm{MeV}$. Charged particles are assumed absorbed in the target. If there are 2 of these neutrons per hadron as defined by eq. (1) then it is found that the equation also describes the neutron emission over a large incident proton energy range. Protons of energy below about $1 \mathrm{GeV}$ will lose significant quantities of energy by ionization in a target and have a finite chance of coming to rest without interacting. A more appropriate quantity to estimate in this case is the neutron emission per proton incident on a target of thickness at least equal to the incident proton range.

The range of a proton of energy $\mathrm{E} \mathrm{GeV}$ (and less than $1 \mathrm{GeV}$ ) can be expressed as:

$$
R=3.5 \lambda_{0} E^{163}
$$

where $\lambda_{0}$ is the nuclear interaction mean free path in the target.

The average number of nuclear interactions per incident proton will be approximately given by: 


$$
N=\left(1-e^{-R / \lambda_{0}}\right)
$$

The mean energy at which an interaction ocçurs in a thick target will be less than the incident energy due to energy losses by ionization; a best estimate of this average interaction energy is $70 \%$ of the incident energy.

Using the above approximations and integrating eq. (1) over all angles, the number of neutrons emitted per incident proton can be determined as a function of proton energy. This is shown in Fig. 4 where the calculations are compared with the number of neutrons per proton in copper determined from a review of experimental data [6]. As can be seen the agreement appears reasonable over the entire energy region down to at least $50 \mathrm{MeV}$.

The secondary neutron flux density, per incident proton, around a copper or iron target at least as thick as the proton range therefore approximates to:

$$
\phi=\frac{\left(1-e^{-3.5 E^{1.63}}\right)}{1.5 R^{2}[\theta+(42 / \sqrt{ } E)]^{2}} \mathrm{n} / \mathrm{cm}^{2} / \text { incident proton }
$$

The value for $R$, the distance from the target in metres has to be sufficient that the target approximates to a point source. The secondary neutron intensity has also to be corrected for any absorption in the target.

The validity of predictions made using the above equation can to some extent be checked by comparson with source terms that have been proposed for estimating lateral shielding for targets. This comparison between the effective dose equivalent at $1 \mathrm{~m}$ from a target perpendicular to the beam direction, determined using eq. (6) and a flux to dose conversion of $4 \times 10^{-14} \mathrm{~Sv} \mathrm{~m}^{2}$ and values estimated from a review of available experimental and theoretical data [7] is shown in Fig. 5. The agreement between predictions using the empirically derived relation and the "best available" values from other independent sources appears to be reasonable at all energies down to $25 \mathrm{MeV}$ incident proton energy. 


\section{CONCLUSIONS}

The empirical relation between incident proton energy and the angular distribution of secondary hadrons produced in an interaction appears to be reasonably valid over a range of incident proton energy from $25 \mathrm{MeV}$ to $1000 \mathrm{GeV}$. At proton energies above about $2 \mathrm{GeV}$ the hadron flux estimated has an energy threshold of $40 \mathrm{MeV}$. For lower energy incident protons the relation is found to represent secondary neutron emission when the flux is increased by a factor of 2 . The relation has been tested where possible against measured fluxes and doses around targets as well as against theoretically derived quantities over the entire proton energy range and no serious discrepancies found. The relation should therefore be valid for estimating the development of the particle cascade in the forward direction from interactions of very high energy protons and be of use for determining the source terms to be used in conjunction with particle buildup and attenuation data, to estimate shielding requirements. The formula presented should also give a good first approximation of fluxes around targets and beam dumps from which induced radioactivity and radiation damage possibilities can be predicted. These predictions should be valid at any emission angle and for incident protons in the energy range $25 \mathrm{MeV}$ to $1000 \mathrm{GeV}$ provided adequate account is taken of ionization losses and absorption of secondaries in thick targets. 


\section{REFERENCES}

1. Hoffmann L., and Sullivan A.H. Studies of the shielding required for the secondary radiation produced by a target in a high energy proton beam (Part V), Nucl. Inst. Meth., 32, 61 (1965).

2. Moore W.H., Source of high energy particles from an internal target in the AGS, Brookhaven National Laboratory Report AGSCD-62 (1966).

3. Levin G.S., Squire P.M. Stapleton G.B., Stevenson G.R., Goebel K., and Ranft J. , The distribution of dose and induced activity around external proton beam targets, in Proc. International Congr. on Protection against Accelerator and space Radiation, CERN 71-16, Vol. 1 (1971), p. 798.

4. Stevenson G.R., Fassò A., Sandberg J., Regelbrugge A., Bonifas A., Muller A., and Nielsen M., Measurements of the hadron yield from copper targets in $200 \mathrm{GeV} / \mathrm{PC}$ and $400 \mathrm{GeV} / \mathrm{c}$ extracted proton beams - An atlas of results obtained, CERN TIS Commission Report TIS-RP/112 (1983).

5. Ranft J., Hadron production in hadron-nucleus and nucleus-nucleus collisions in a dual Monte Carlo multichain fragmentation model, Phys. Rev. 37(7), 1842 (1988).

6. Tesch K., A simple estimation for the lateral shielding for proton accelerators in the energy range 50 to $1000 \mathrm{MeV}$, Rad. Prot. Dosimetry 11(3), 165 (1985).

7. Stevenson G.R., Shielding at proton energies of less than $3 \mathrm{GeV}$, CERN TIS-RP/IR/86-08 (1986). 


\section{FIGURE CAPTIONS}

Fig. 1. Comparison of the empirical relation (solid line) with hadron flux ( $\mathrm{E}>40 \mathrm{MeV}$ ) measured at $1 \mathrm{~m}$ from a copper target,

a) for protons of energy 8 and $24 \mathrm{GeV}$ incident on a $5 \mathrm{~cm}$ copper target (in which $22 \%$ of the protons are assumed to interact), and b) for 225 and $400 \mathrm{GeV}$ protons.

Fig. 2. Hadron flux per interaction at $1 \mathrm{~m}$ in the forward and sideways direction as a function of interacting proton energy.

Fig. 3. Estimated total number of hadrons emitted per interaction on a copper or iron target as a function of proton energy. Also indicated is the number of hadrons calculated using independent Monte Carlo computations.

Fig. 4. Estimated number of neutrons emitted per incident proton in copper (solid line) compared with independently determined values.

Fig. 5. Estimated dose equivalent per incident proton at $1 \mathrm{~m}$ and $90^{\circ}$ to a target of length equal to the range of the incident proton as a function of the incident proton energy compared with independently determined values. 


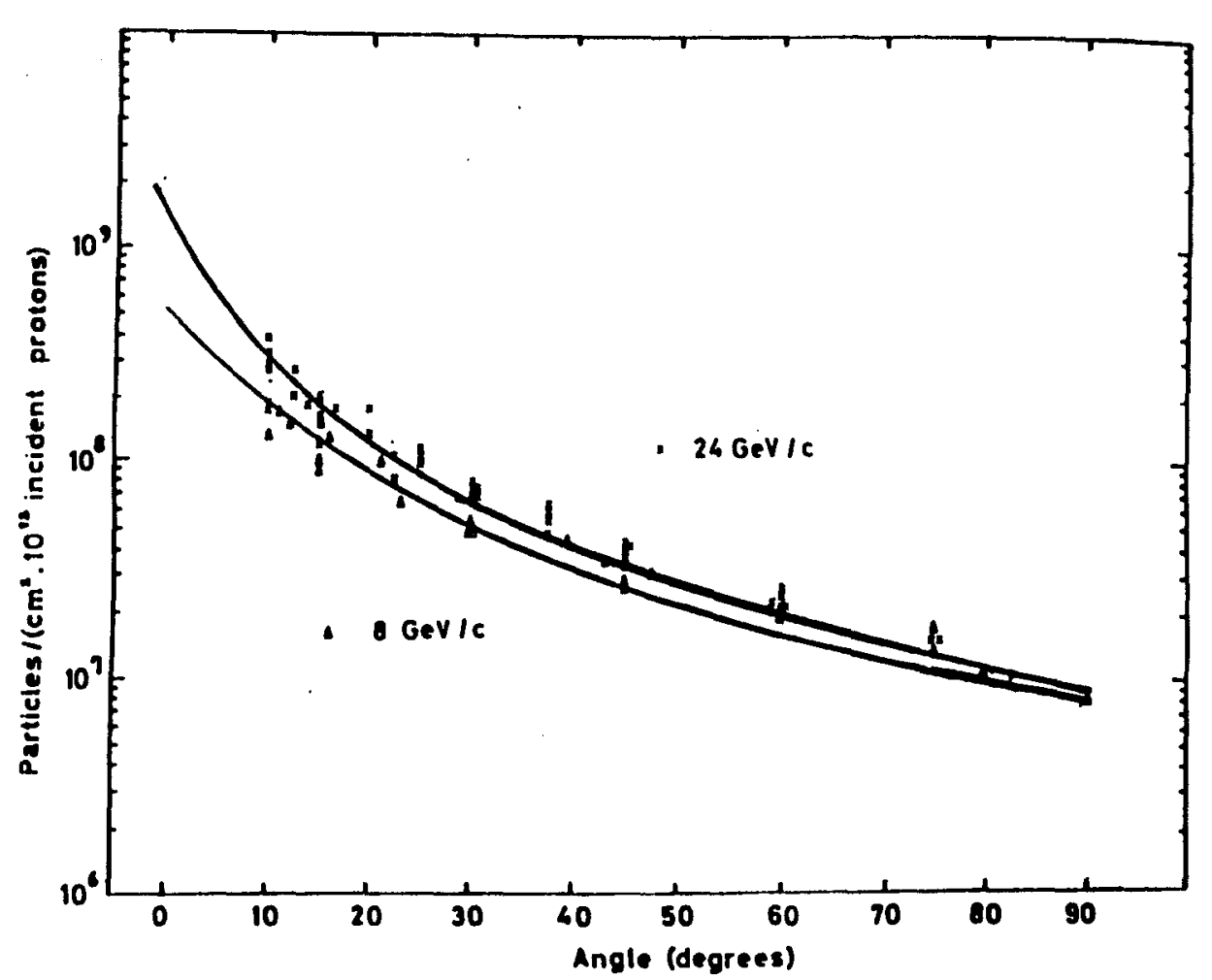

Fig. $1 a$

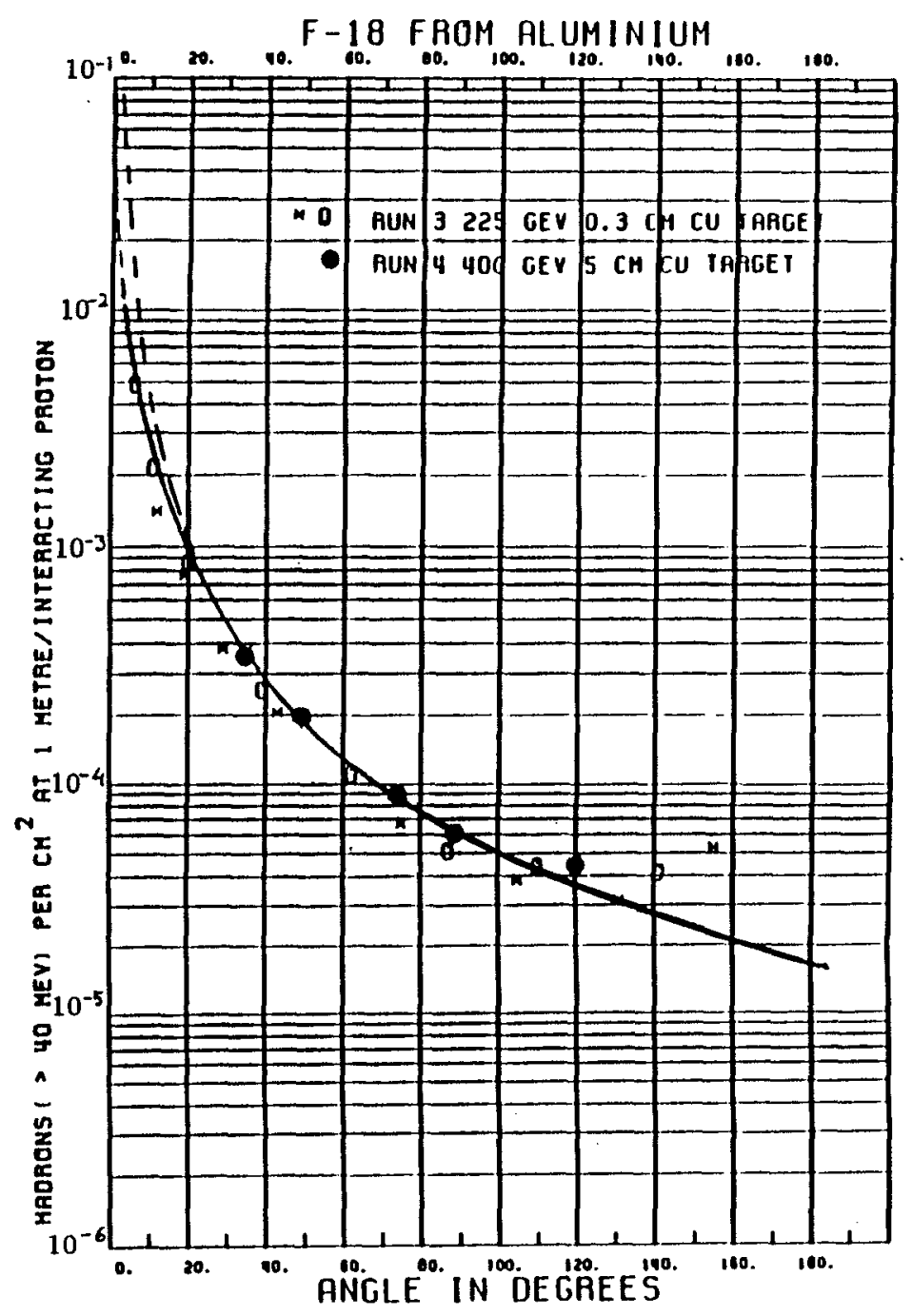

Fig. 1b 


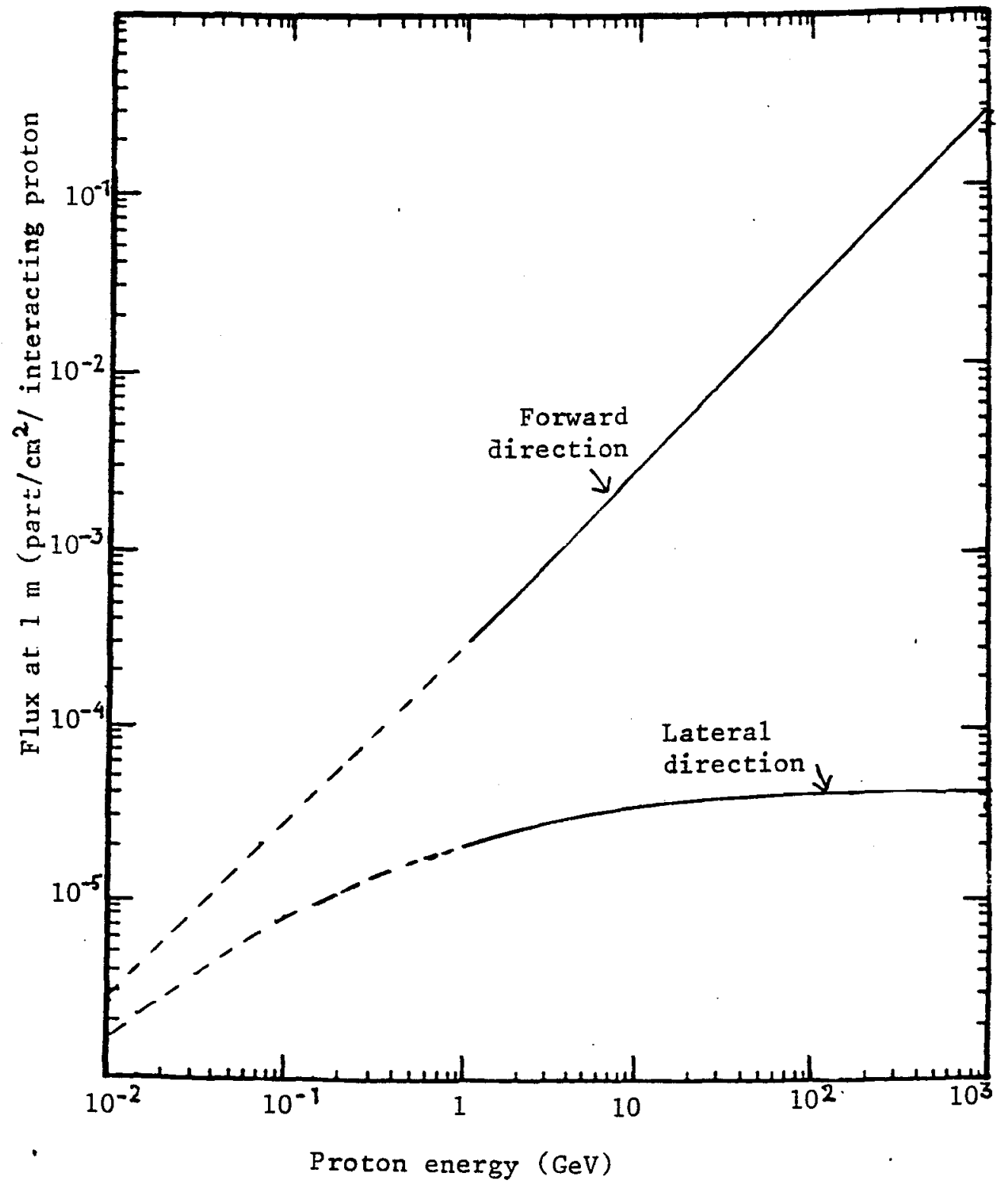

Fig. 2 


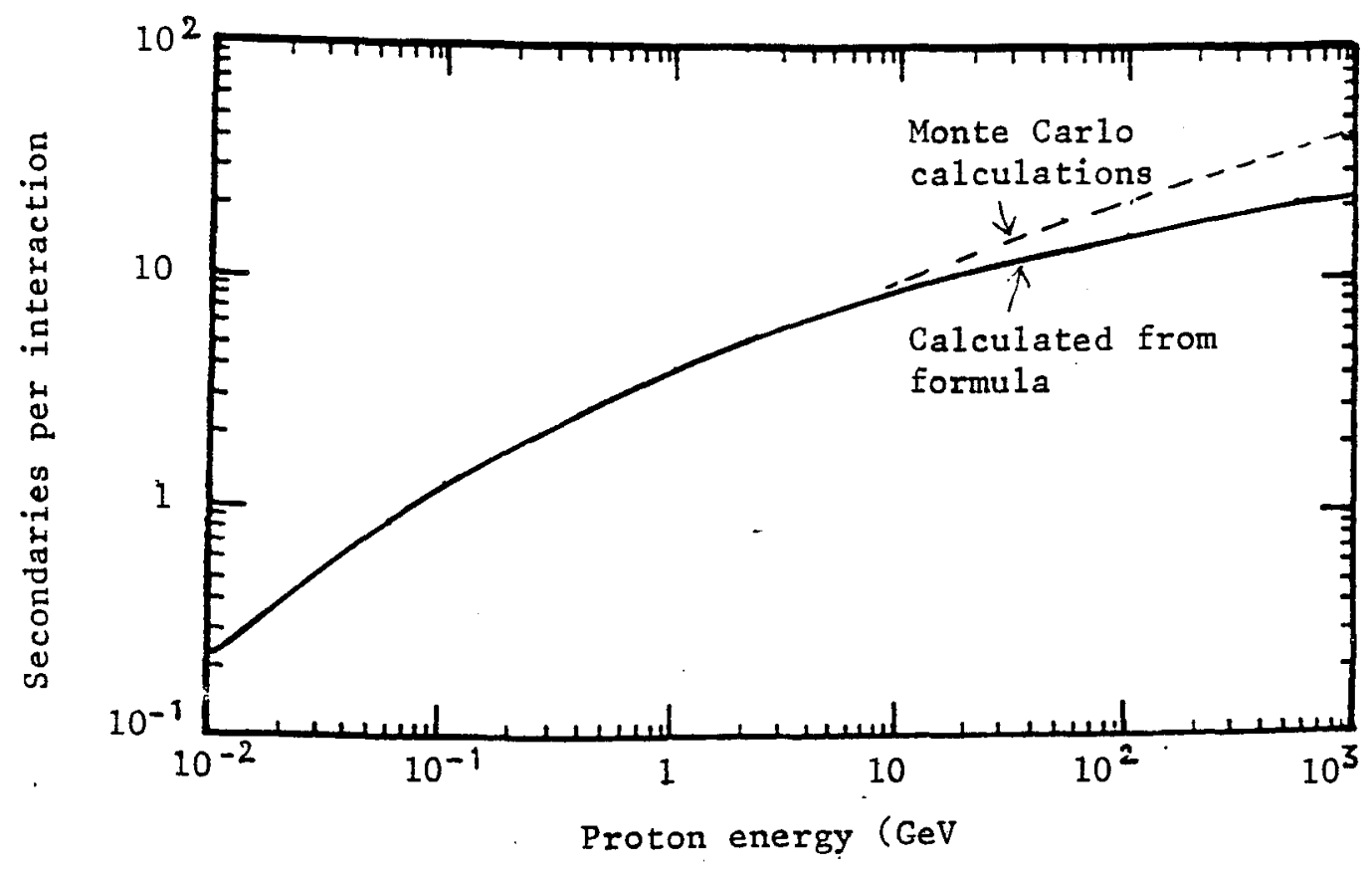

Fig. 3 


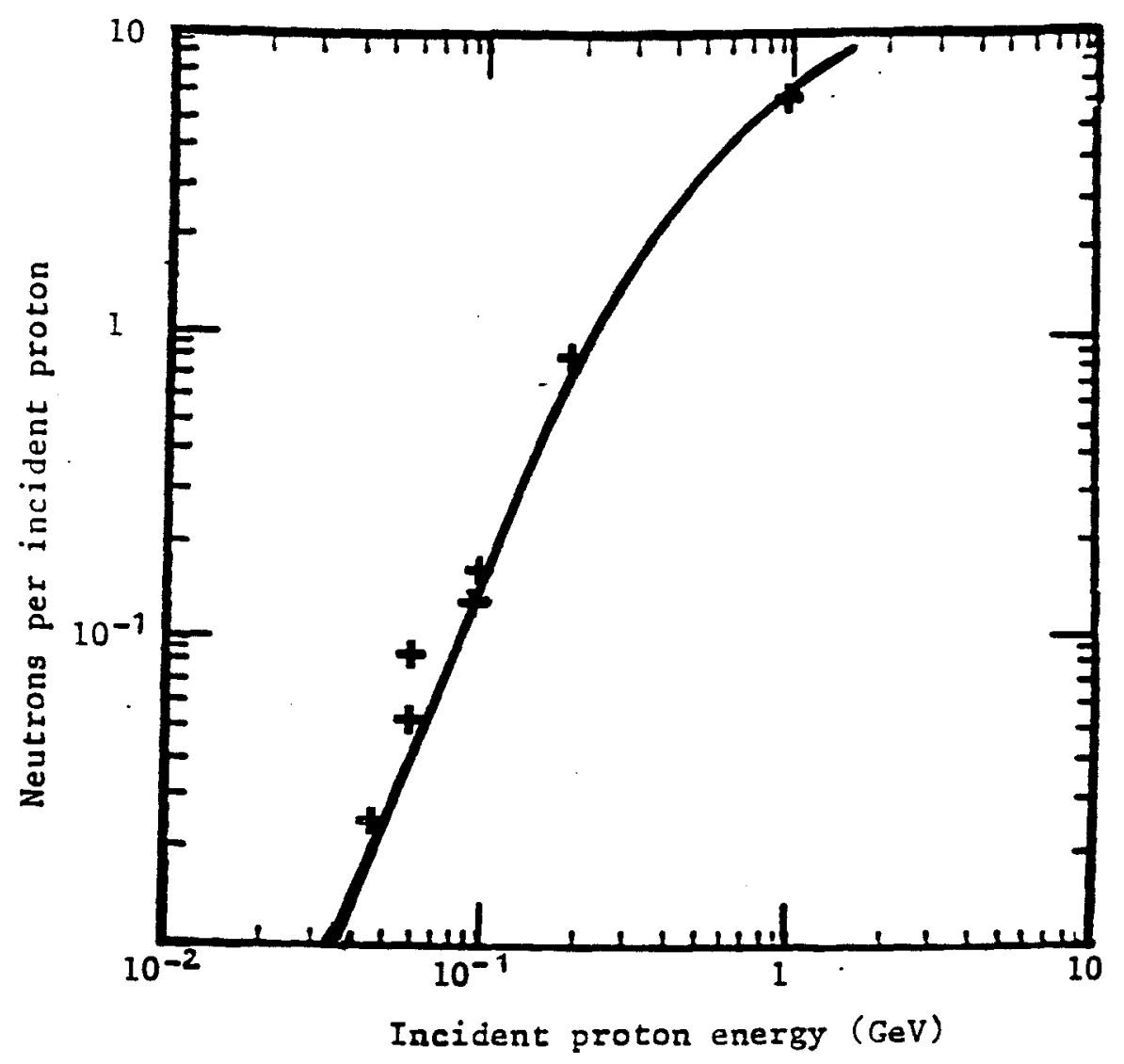

Fig. 4 


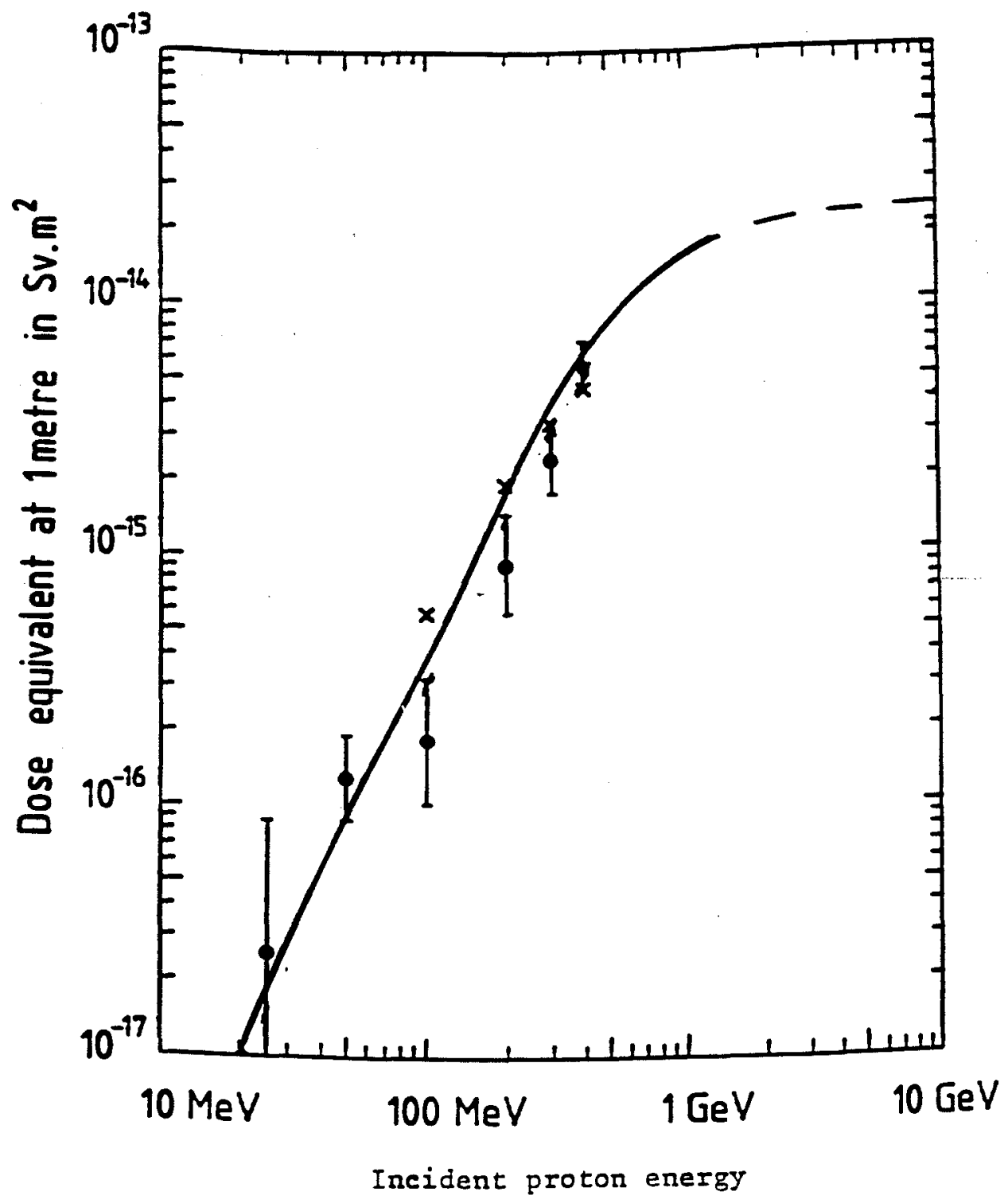

Fig. 5 